\title{
6. The Super TB Experiment: Evolution and Resolution of an Experiment with Dual-Use Concerns
}

\author{
Nancy Connell
}

\section{Introduction}

Tuberculosis (TB) is a devastating disease that leads to 10 million deaths per year worldwide. ${ }^{1}$ Despite enormous advances in TB research in recent years, the surge of drug-resistant strains and deadly synergy with the HIV virus have threatened to destabilise gains made in its control. During my career as a microbial geneticist, I have studied the physiology of Mycobacterium tuberculosis, the causative agent of TB, and its interaction with the human macrophage, its primary host cell. The major function of the macrophage is to engulf and destroy invading organisms, but many pathogens, such as $\mathrm{TB}$, have developed intricate and clever ways to avoid or subvert these host defences.

This chapter discusses how a classical approach to asking a very basic question about microbial pathogenesis can lead to a surprising result and a dual-use dilemma. The elimination of a single gene from $M$. tuberculosis created a strain with increased virulence when measured in a model system (in vitro) but not during animal infection (in vivo).

\section{Experiments of concern}

The concept of dual-use research - that certain kinds of experimental endeavours might be used for malignant purposes despite their original beneficent intenthas entered the biomedical lexicon. ${ }^{2}$ My personal interest in the matter grew out

\footnotetext{
$1<$ http://www.who.int/tb/publications/global_report/2010/en/index.html $>$.

2 National Research Council and the American Association for the Advancement of Science (NRC/AAAS) 2009, A Survey of Attitudes and Actions on Dual Use Research in the Life Sciences: A Collaborative Effort of the National Research Council and the American Association for the Advancement of Science, Washington, DC; American Association for the Advancement of Science (AAAS) 2009, Building the Biodefense Policy Workforce, American Association for the Advancement of Science, Washington, DC; American Association for the Advancement of Science (AAAS) 2008, Professional and Graduate-Level Programs on Dual Use Research and Biosecurity for Scientists Working in the Biological Sciences, American Association for the Advancement of Science, Washington, DC; Atlas, R. and Dando, M. 2006, 'The dual-use dilemma for the life sciences: perspectives, conundrums, and global solutions', Biosecurity and Bioterrorism, vol. 4, no. 3, pp. 1-11.
} 
of a longstanding interest in biological weapons and arms control beginning in the 1980s. At the time, many scientists were contributing to the development of a Verification Protocol for the Biological and Toxin Weapons Convention (BTWC). ${ }^{3}$ Part of that commitment included the responsibility of scientists to be aware of the societal impact of their work and to communicate that impact to the public.

In response to concerns about dual-use research, a number of advocacy groups started up at this time such as Science for the People and the Committee for Responsible Genetics (now the Council for Responsible Genetics). Simultaneously, scientists and policy analysts realised that the potential for harm caused by naturally occurring organisms and toxins could be easily surpassed by the creative development of recombinant agents expressing altered or novel virulence characteristics. Commonly available genetic technologies in laboratories improved, allowing for a deeper understanding and dissection of the mechanisms of infection and virulence.

As the Verification Protocol fell by the wayside in the autumn of 2001, one month before the anthrax mailings in the United States, interest in strengthening the BTWC took on new directions. One of those was an interest in how knowledge and techniques being generated as part of peaceful work in the life sciences could facilitate the deliberate spread of disease - what is now known as 'dual use'. A list of the experiments considered to be of dual-use concern was published in several reports shortly after the events of 2001. This included the landmark Fink Report. ${ }^{4}$ These lists included several approaches to creating novel mutants of existing pathogens, such as: 1) developing antibiotic-resistant strains, 2) expanding host range, 3) increasing transmissibility, and 4) altering virulence.

To understand a biological system, one of the first approaches is to perturb the system and observe the effects of that manipulation. In a genetic analysis, the approach is to alter or even eliminate the function of a gene and observe the effects of that alteration on the biological process. By integrating information from a number of different alterations of a single gene, its role or function in a system or pathway can be deduced. In the case of disease-causing organisms, each of the 'experiments of concern', listed above, fits into this classic approach to understanding biological function

- antibiotic-resistant strains are selected and characterised to understand the mechanism of action of an antibiotic, and the identification of the target will allow development of more effective antibiotics directed to that target

\footnotetext{
3 Zilinskas, R. A. 1998, 'Verifying compliance to the biological and toxin weapons convention', Critical Reviews in Microbiology, vol. 24, pp. 195-218.

4 National Research Council 2004, Biotechnology Research in An Age of Terrorism, The National Academies Press, Washington, DC.
} 
- host range mutants are developed to understand the mechanism of host range restriction and to develop methods of blocking host-pathogen interaction, the first step of infection

- transmissibility mutants can be created to understand the mechanisms that control transmissibility, to develop methods of reducing transmissibility

- altering virulence can be used to identify the genes encoding virulence factors, and interfering with these factors that may limit infection and pathogenesis of disease.

Therefore, any comprehensive genetic analysis of virulence will eventually be confronted with the possibility of an 'experiment of concern'. The question for researchers is whether to continue to pursue the line of inquiry or to find an alternative approach. I now want to consider these issues in relation to my own work.

\section{The experiment: Nutrient transport mutants of Mycobacterium tuberculosis}

Microbes use small molecules such as sugars and amino acids to survive, and pathogens that live in their host's cells have a multitude of ways to acquire these nutrients from their environment. In the case of $M$. tuberculosis, their environment is the inside of mammalian white blood cells, where they replicate quite happily; indeed, the bacterium's method of pathogenesis is to reside within the very cells designed to protect the host against them! ${ }^{5}$

Our experimental approach was to create mutants of $M$. tuberculosis that were defective in uptake of a certain class of small nutrients called peptides. These are short (2-6) chains of amino acids that are transported by efficient transport systems called ABC transporters. ${ }^{6}$ We were able to construct mutants of $M$. tuberculosis lacking the genes that encode one of these transporters.

Simultaneously, we also discovered that a small tri-peptide called glutathione had the unusual and unexpected property of being toxic to M. tuberculosis. $^{7}$ This meant that in the presence of this small peptide, M. tuberculosis could not grow. The concentration of glutathione in mammalian cells varies considerably but is usually quite high; under some conditions, the concentration is much higher than the toxic levels that kill $M$. tuberculosis. We wondered how this

\footnotetext{
5 Pieters, J. 2008, 'Mycobacterium tuberculosis and the macrophage: maintaining a balance', Cell Host and Microbe, vol. 3, pp. 399-407.

6 Detmers, F. J. M., Lanfermeijer, F. C. and Poolman, B. 2001, 'Peptides and ATP binding cassette peptide transporters', Research in Microbiology, vol. 152, pp. 245-58.

7 Green, R. M., Seth, A. and Connell, N. D. 2000, 'A peptide permease mutant of Mycobacterium bovis BCG resistant to the toxic peptides glutathione and S-nitrosoglutathione', Infection and Immunity, vol. 68, pp. 429-36.
} 
situation could have developed: that a bacterium that spends its entire life cycle in mammalian cells does so in the presence of a small molecule, glutathione, that is toxic! We characterised this toxicity, and showed that in mouse-derived cells, when the glutathione levels were high, M. tuberculosis did not grow well, but when the glutathione levels were low, the bacteria grew very efficiently. ${ }^{8}$

We were, however, now at a crossroads. First, we showed that glutathione is toxic to $M$. tuberculosis, and we found glutathione at toxic levels in all mammalian cells. Second, we had created a mutant of M. tuberculosis in the laboratory that was lacking a peptide transporter and incapable of accumulating small peptides, including glutathione. Therefore, this mutant should have been resistant to the toxic effects of glutathione. We showed that this was indeed the case.

But if glutathione is toxic to M. tuberculosis and present in all mammalian cells then it might play a natural role in limiting M. tuberculosis growth. This was our hypothesis. The logical next step was to see whether our mutant strain survived better than its normal parent strain during intracellular growth in white blood cells in tissue culture. It did. In fact, the mutant strain grew to three to five times higher levels than normal parent strains when inoculated and grown in cultured macrophage cell lines. Our experimental approach following a logical series of steps had led us to create a hyper-virulent mutant of M. tuberculosis. We had generated a strain that survived in host cells better than its normal parent strain, as a result of the deletion of a single gene encoding one component of a basic nutrient transport system. Interestingly, the dual-use implications of our work were brought to my attention only during a discussion of that issue with members of the Controlling Dangerous Pathogens Project at the Center for International and Security Studies at Maryland (Elisa Harris and colleagues). Apparently, I had partitioned my thinking about these matters, and separated the experiment itself from the possible ethical concerns associated with it, despite my own decades-long involvement with the issue of biological weapons and the responsibility of scientists in preventing a biological arms race.

\section{Virulence factors}

Defining virulence is not a simple matter. By a general and superficial definition, virulence is the degree of 'pathogenicity' of a biological agent, and pathogenicity

8 Venketaraman, V., Dayaram, Y. K., Amin, A. G., Ngo, R., Green, R. M., Talaue, M. T., Mann, J. and Connell, N. D. 2003, 'Role of glutathione in macrophage control of mycobacteria', Infection and Immunity, vol. 71, pp. 1864-71; Venketaraman, V., Dayaram,Y. K., Talaue, M. T. and Connell, N. D. 2005, 'Glutathione and nitrosoglutathione in macrophage defense against Mycobacterium tuberculosis', Infection and Immunity, vol. 73, pp. 1886-9. 
is the ability of an organism to cause disease. Both terms are relative and highly dependent on context. Furthermore, virulence and pathogenicity are frequently used interchangeably.

Myriad factors contribute to the virulence of an infectious agent. Virulence factors that cause severe damage to the host are called toxins. Some toxins act directly on the host cell such as listeriolysin, which punches holes in the membranes of host cells and breaks them open. ${ }^{9}$ Many viruses reproduce to such high numbers in cells - grabbing bits of the host cell membranes as they gothat the cells just break apart. ${ }^{10}$ Other toxins act directly on host cells to alter their function severely. For example, one component of anthrax toxin destroys the proteins that orchestrate the immune response and cause swelling in the tissues surrounding the site of infection, while another component inactivates a small protein, called G-protein, which regulates the movement of ions across membranes; its inactivation leads to severe oedema. ${ }^{11}$ Other toxins block protein synthesis, so the cell can no longer carry out normal functions (diphtheria toxin), ${ }^{12}$ or interfere with neurotransmitter activities, as with the clostridial toxins, botulinum toxin and tetanus. ${ }^{13}$ In some, but not all, cases elimination of the gene encoding the toxin will render the bacterium completely harmless ('avirulent'); this is true with Clostridium botulinum, the causative agent of botulism. Conversely, expression of the gene encoding the botulinum toxin in an alternative bacterial host would lead to the creation of a very dangerous agent. Ken Alibek, a former Soviet bioweapons researcher, claimed that the Soviet offensive program devoted considerable effort to splicing the botulinum toxin genes into other bacteria for delivery. ${ }^{14}$

But in the case of Yersinia pestis (plague), ${ }^{15}$ for example, and Burkholderia mallei (glanders) ${ }^{16}$ - both select agents - there are many small, secreted virulence factors that work together to damage the host; elimination of one or two of the genes encoding the complex array of 'effectors' will not lead to inhibition of virulence, rather, just defects in the ability to cause disease ('hypo-virulent',

9 Schnupf, P. and Portnoy, D. A. 2007, 'Listeriolysin O: a phagosome-specific lysin', Microbes and Infection, vol. 9, pp. 1176-87.

10 Kaminskyy, V. and Zhivotovsky, B. 2010, 'To kill or be killed: how viruses interact with the cell death machinery', Journal of Internal Medicine, vol. 267, pp. 473-82.

11 Moayeri, M. and Leppla S. H. 2009, 'Cellular and systemic effects of anthrax lethal toxin and edema toxin', Molecular Aspects of Medicine, vol. 30, pp. 439-55.

12 Iglewski, W. J. 1994, 'Cellular ADP-ribosylation of elongation factor 2', Molecular and Cellular Biochemistry, vol. 138, pp. 131-3.

13 Popoff, M. R. and Bouvet, P. 2009, 'Clostridial toxins', Future Microbiology, vol. 4, pp. 1021-64.

14 Alibek, K. and Handleman, S. 1999, Biohazard, Random House, New York.

15 Viboud, G. I. and Bliska, J. B. 2005, 'Yersinia outer proteins: role in modulation of host cell signaling responses and pathogenesis', Annual Review of Microbiology, vol. 59, p. 689.

16 Sun, G. W. and Gan, Y. H. 2010, 'Unraveling type III secretion systems in the highly versatile Burkholderia pseudomallei', Trends in Microbiology, vol. 18, pp. 561-8. 
or 'attenuated'). By the same token, expression of these individual effector molecules in other bacteria would not likely lead to creation of a more virulent organism, without the context of the entire genome for full virulence.

\section{The many faces of virulence?}

The experimental models used to identify and characterise these virulence factors include bacterial culture conditions, infection of mammalian cells in culture and small animal/non-human primates. ${ }^{17}$ Each of these approaches has its limitations and can affect the way in which overall virulence is expressed. Indeed, the terms hypo-virulence, hyper-virulence, avirulence and antivirulence ${ }^{18}$ are all common in the medical literature. Yet the use of each of these terms must be considered in its context.

Returning to the experiments described above, we had identified a mutant strain of $M$. tuberculosis that exhibited hyper-virulent characteristics only in the context of the cell culture model - that is, mammalian cells grown in petri dishes in incubators. We were at another crossroads: should we go ahead and perform an experiment in an animal infection model of TB? Our laboratory was under some pressure from funding agencies to demonstrate that glutathione resistance was relevant in the larger scale model of mouse infection, which is more closely relevant to human disease than the tissue culture model we used for our more preliminary experiments.

We decided that the experiment was worth performing. We felt that if we could show the relevance of our observation to TB disease in the whole animal, we could convince the reviewers of our grant proposals that manipulation of the glutathione system might be exploited to limit TB disease. Fully mindful of the safety risks, we consulted our Institutional Biosafety and Institutional Animal Use and Care committees, and we performed a risk analysis that weighed the risks and benefits of the experiment. We concluded that knowing the answer to this question was important for the project's direction and that our biosafety plans were sufficient to carry out the experiment safely. Among our discussion points was the observation that there is a high degree of variability among clinical strains of $M$. tuberculosis, and our new strain was well within the range of virulence observed in cell culture models. We were not dealing with a strain of unusually extreme virulence. So we designed and executed a mouse infection experiment under biosafety level three (BSL3) containment conditions with

17 O'Toole, R. 2010, 'Experimental models to study human tuberculosis', Advances in Applied Microbiology, vol. 71, pp. 75-89.

18 Casadevall, A. and Pirofski, L. 2002, “'Anti-virulence" genes-further muddling the lexicon?' Trends in Microbiology, vol. 111, pp. 413-14. 
appropriate safety and security measures. We found that there was no difference in the survival or replication rate of the mutant compared with the normal M. tuberculosis strain (unpublished observations). Thus, the hyper-virulent behaviour we observed in the tissue culture model was not carried through in an experimental system closer to the human disease state.

Our increased awareness of the dual-use implications of altering virulence significantly impacted on our deliberations concerning additional testing of our 'hyper-virulent' mutant of $M$. tuberculosis. On the one hand, we were under considerable pressure from our grant reviewers to demonstrate hyper-virulence in the mouse model. If we did not demonstrate hyper-virulence then they might not consider funding out project. On the other hand, our deliberations in the laboratory about the nature of virulence and pathogenicity increased our appreciation of the complexity of infectious potential and the inherent dual-use nature of our research. These are the kinds of careful discussions that we would urge scientists to have at many stages of their work. 